\title{
Skizze einer Geschichte der angewandten Geographie in der Schweiz
}

\author{
Ernst Winkler
}

Der Unterricht (in Geographie) scheint noch sehr mangelhaft zu sein. Nichtsdestoweniger ist es gerade sie, von der man in allen nur möglichen Verhältnissen den nützlichsten Gebrauch zu machen imstande ist.

J. Kant

Die sogenannte angewandte ${ }^{1}$ oder praktische Geographie hat offenbar auch in der Schweiz Fuß gefaßt. Jedenfalls taucht der Name immer häufiger in der Fach- und Tagespresse auf. Nicht selten hat es dabei den Anschein, als ob es sich um eine neue Errungenschaft mit dem Zweck handle, der Geographie vermehrte Verwendungsmöglichkeiten zu verschaffen, ja ihr erst eigentlichen Sinn zu verleihen. Nun war von keinem Geringeren als von A. Hettner, dem bisher bedeutendsten Methodologen der Geographie, schon vor Jahrzehnten wiederholt festgestellt worden, daß diese Disziplin seit den Anfängen und bis in die neuere Zeit hinein "ganz im Dienste der praktischen Zwecke des Lebens gestanden" habe 2 . Und lange vor ihm hatte der Philosoph J. Kant sogar ausdrücklich versichert, daß man gerade von ihr «in allen nur möglichen Verhältnissen des Lebens den nützlichsten Gebrauch zu machen imstande" sei ${ }^{3}$. "Es war», sagte Hettner demgegenüber in seinem Werk "Die Geographie, ihre Geschichte, ihr Wesen und ihre Methoden" (1927), «das große Verdienst Karl Ritters, daß er den praktischen Interessen gegenüber die reine Wissenschaft zur Geltung brachte, und seit den letzten Jahrzehnten des vorigen Jahrhunderts hat sich die Geographie als Wissenschaft durchgesetzt»4. In Tat und Wahrheit vermochte sie sich allerdings nur bedingt «durchzusetzen». Hierüber dürften sich die Geographen sicher einig sein 5 . Die Versuche, ihrer Disziplin vermehrte Anwendungen zu verschaffen, die nicht zuletzt in Forderungen nach Lehrstühlen für angewandte Geographie an den Hochschulen gipfelten (und die bekanntlich bereits teilweise Verwirklichung fanden), sind deshalb durchaus begreiflich. Andrerseits ist nicht zu übersehen, daß die Geographie doch verschiedentlich Eingang ins praktische Leben gefunden hat. Im folgenden sei versucht, dies am Beispiel der Schweiz zu zeigen.

Die älteren Entwicklungsperioden dürfen hierbei wohl übergangen werden, obgleich sich - etwa anhand der "Geschichte der Physischen Geographie der Schweiz bis 1815 » von B. Studer ${ }^{6}$ zeigen ließe, daß, was Hettner vor Jahren allgemein gesagt hatte, auch für unser Land Geltung besitzt. Um die aktuelle Geographie nun auf ihrem historischen Hintergrund zu beurteilen, genügt es indessen, an den früheren Altmeister der Schweizer Geographie, J. Früh (1862-1938), anzuknüpfen. Ursprünglich Naturwissenschafter, wandte er sich bekanntlich später der Erdkunde zu und schenkte ihr die erste moderne umfassende Geographie der Schweiz, darin ausdrücklich festhaltend, daß sie «weder die einzelnen Dinge an sich wie die beschreibenden Naturwissenschaften, noch den Menschen ..., sondern die Wechselbeziehungen der verschiedenen ...... Erscheinungen in Bezug auf die Erdoberfläche» zu betrachten habe ${ }^{7}$. Wiederholt erhärtete er außerdem die Notwendigkeit, seine Disziplin in den Dienst der Mitbürger, des Schweizervolkes und der Eidgenossenschaft zu stellen. Sie war für ihn also durchaus zweckbestimmt, erhielt ihren Sinn nur durch ihre Anwendbarkeit.

Von ihm führen die Fäden «angewandter» Geographie ununterbrochen zur Gegenwart. An deren Schwelle steht, als wohl ausschließlichster «angewandter» Geograph der Folgezeit, Hans Bernhard (1888-1942). Anfänglich Agronom, bildete er sich nach seinem Fachstudium zum ausgesprochenen Agrargeographen aus. Bereits in seiner grundlegenden Abhandlung "Die Agrargeographie als wissenschaftliche Disziplin» $(1915)^{8}$ gab er zu erkennen, daß ihm die Forschung ausschließlich Mittel zum Zwecke optimaler menschlicher Wohlfahrt sei. Damit verlieh er der unbestreitbaren Tatsache Ausdruck, daß alle Wissenschaft, als Element der Kultur nur als deren Organ sinnvoll ist. Der Erste Weltkrieg trieb ihn zur Verwirklichung seiner theoretischen Ansichten. Er «lehrte ihn einsehen, daß es ein Fehler war, wenn in den letzten Jahrzehnten der heimische Nährfruchtbau den Einflüssen des Weltmarktes preisgegeben, der Vereinseitigung unserer Bodenkultur zur Viehzucht nicht gewehrt wurde. Er brachte die Erkenntnis, daß ein Land, wenn es Versorgungsnöten nicht ausgesetzt sein will, gut daran tut, die Zahl der Nahrungsschaffenden und der Nahrungszehrenden in einem gewissen Gleichgewicht $\mathrm{zu}$ halten, der Übersättigung mit Industrie vorzubeugen. Und die Schwierigkeiten der Lebensmittelbeschaffung für die Städte wiesen mit drohendem Finger darauf hin, daß das gedankenlose Aneinanderreihen vonWohnstätten beimAusbau der großen Orte ein Unglück war; es fehlte den Massen- 
siedlungen an der harmonischen Beziehung zum ernährenden Boden, die allein ein ungefährdetes, dauerndes Bestehen gestattet ... Bei dieser Gelegenheit tauchte auch bei uns der Begriff der inneren Kolonisation auf ... Was wollen die (mit ihr verknüpften) Vorkehrungen? Doch einfach das, einer größeren Anzahl Menschen in unserem Lande Nähr- und Wohnraum schaffen, und in besserer Weise als er bis anhin vorhanden war $^{9}$."

Mit dieser Umschreibung weitete Bernhard den üblichen Begriff Innenkolonisation zur umfassenden Landesplanung und -gestaltung aus, deren Ziel die bestmögliche Aufeinanderabstimmung aller Interessen an der Landschaft: der Interessen des Wohnens, Arbeitens, Erholens und Verkehrens mit dem ausschließlichen Ziel der individuellen und gesamtsozialen Wohlfahrt bei optimaler Schonung dieser Landschaft, des menschlichen Lebensraumes ist. Bernhard wurde so zu einem der ersten und aktivsten Begründer der modernen schweizerischen Landesplanung und damit zugleich zu einem "Erneuerer» der "angewandten» Geographie in unserem Lande. In der Folge gelangen ihm bedeutsamste nationale und regionale Verwirklichungen landesplanerischer Art: Meliorationen, Siedlungskorrekturen und -neugründungen usw.10. Als tragendes Instrument diente ihm die von ihm 1918 gegründete «Schweizerische Vereinigung für Innenkolonisation und industrielle Landwirtschaft». Immer blieb ihm aber seine geliebte Agrargeographie der Schlüssel aller seiner Aktionen. Noch kurz vor seinem für sein Land viel zu frühen Tod, 1939, gab er seiner Überzeugung in einem Vortrag vor der GeographischEthnographischen GeselIschaft Zürich mit den Worten Ausdruck: "Es war mir darum zu tun, am Beispiel der schweizerischen Agrarverhältnisse darzutun, daß geographische Gesichtspunkte in der Einflußnahme auf die Weiterentwicklung bestehender wirtschaftlicher Verhältnisse praktisch gut verwendet werden können. In dieser angewandten Geographie betätigen wir Heimatkunde bester Art: wir helfen aktiv mit, unseren vaterländischen Boden ... als Nähr- und Wohnraum bis zum menschenmöglichen Letzten $z u$ erschließen»11.

Gewiß nicht zumindest als Wirkung der Tätigkeit H. Bernhards blieb die Geographie auch in der folgenden Zeit mit der Praxis eng verbunden. Da ihre Pflege vornehmlich bei den Hochschulinstituten konzentriert ist, darf wohl die Skizze der seitherigen angewandten Geographie mit ihnen beginnen. Damit soll jedoch weder eine Dominanz derselben noch eine Priorität gegenüber privaten oder individuellen Bemühungen beansprucht werden.

Es sei erlaubt, Zürich und die Eidgenössische Technische Hochschule vorwegzunehmen. Auch in ihrem geographischen Institut rissen seit Früh die Beziehungen zur Praxis nie ab, wiewohl die unmittelbaren Nachfolger, F. Machatschek und O. Leh- mann, wohl namentlich infolge ihrer ausländischen Herkunft, dies weniger zum Ausdruck zu bringen vermochten. Vom Bemühen des zuletzt Genannten, Geographie lebensnah zu gestalten, zeugt jedenfalls etwa die in Zusammenarbeit mit H. Bernhard verfaßte Abhandlung «Der Wandel der Landschaft im oberen Sihlgebiet» (1938)12. In H. Gutersohn fand sodann, seit 1941, der Kontakt zwischen geographischer Theorie und Praxis einen ebenso initiativen wie fruchtbaren Betreuer. Schon vor seiner Wahl zum Ordinarius der ETH hatte er sich mit Anwendungsfragen seiner Disziplin beschäftigt wie z. B. sein Vortrag "Geographische Gesichtspunkte für eine Stadtgründung in Brasilien» (1940) zeigt ${ }^{13}$. Unmittelbar nach der Übernahme des Lehrstuhls stellte er sich in den Dienst der damals aufkommenden schweizerischen Landesplanung. Er förderte sie nicht nur maßgebend als Mitbegründer des Planungsunterrichts an der ETH und des hiefür geschaffenen Instituts (1943). Er lieh ihr auch durch unmittelbare praktische Tätigkeit, als Mitglied der Schweizerischen Vereinigung für Landesplanung, der er lange und teils sehr schwierige Jahre als Präsident vorstand, wertvollste Hilfe. Sie fand in zahlreichen Initiativen, Publikationen, Gutachten und Vorträgen Ausdruck. Daß er in diesem Rahmen zugleich den Landschaftsschutz und die Landschaftspflege wirksam unterstützte, bedarf kaum besonderer Hervorhebung. Wohl aber gebührt seiner Tätigkeit als Präsident der Alpenstraßenkommission, welche den Bau der Nationalstraßen mit vorzubereiten hatte und als Präsident der Schweizerischen Koordinationskommission für die Technische Hilfe (an Entwicklungsländer) spezieller Erwähnung, da darin, abgesehen von der persönlichen Wertschätzung auch die Anerkennung seines Faches Geographie Ausprägung empfing. Nicht zuletzt ist im Gesamtzusammenhang seines fundamentalen Hauptwerkes "Geographie der Schweiz» zu gedenken, die bewußt auch als Grundlage der Regional- und Landesplanung konzipiert wurde ${ }^{14}$ und in welcher auch die "quantitative» Geographie, die Gutersohn während seiner Tätigkeit besonders förderte, Berücksichtigung erfuhr.

Dem Schreibenden, der seit 1925 als Mitarbeiter von $\mathrm{H}$. Bernhard - z. B. zusammen mit seinem Studienkollegen W. Kündig beim Landerwerb für das Sihlkraftwerk - Gelegenheit hatte, "praktische" Geographie zu lernen, war vergönnt, das Wirken von H. Gutersohn als Assistent und Kollege zu begleiten. Er gedenkt umso dankbarer der Anregungen, die er hieraus empfing, als für ihn der Sinn aller Wissenschaft ausschließlich in der Möglichkeit ihrer Anwendung besteht.

Nicht minder als im Geographischen Institut der ETH wurde an dem der Zürcher Universität, an dem Bernhard längere Jahre als Dozent geamtet hatte, angewandte Geographie gepflegt. Sowohl 
H. J. Wehrli (1871-1945), als auch O. Flückiger (1881-1942) ließen sich angelegen sein, die Geographie als "Wissenschaft für das Leben» zu lehren, wobei sie nicht wenig auf ihrem Vorgänger O. Stoll (1849-1922) aufbauten. Vor allem aber bemühte sich seit 1940 ihr Nachfolger, H. Boesch, der anschließend an längere Amerikaaufenthalte schon in den dreißiger Jahren auf die Geographie als Grundlage der Landesplanung hingewiesen hatte, deren Fruchtbarkeit unter Beweis zu stellen. Mit eigenen Arbeiten etwa über Geographie und Planung in den USA und England (1944) ansetzend15, konnte er bald tüchtige Schüler für seine Ideen gewinnen. Indem er H. Carol, dessen "Wirtschaftsgeographische Karte der Schweiz» (1946) ${ }^{16}$ noch jetzt als willkommene Planungsgrundlage Verwertung findet, als Mitarbeiter und Kontaktmann zur kantonalen Regionalplanung einsetzte, gelang ihm, mit diesem zusammen die Einschaltung der Geographie in die Praxis in einem Maße, wie dies vor dem kaum möglich erschienen war. Wegweisende - leider nicht veröffentlichte - Strukturatlanten für die Kantone Zürich und Aargau bildeten hiefür gleicherweise positive Zeugnisse wie die Mitwirkung bei grundlegenden Gutachten der Planung. Das Buch "Städte wie wir sie wünschen»17 von $\mathrm{H}$. Carol und $\mathrm{M}$. Werner, an welchem weitere Geographen, meist Schüler von H. Boesch (G. Gensler, R. Merian, O. Wernli u. a.) mitgearbeitet hatten und das nicht zuletzt unter dem Protektorat von diesem und $\mathrm{H}$. Gutersohn entstand, darf noch heute als Markstein der schweizerischen Landesplanungsliteratur gelten. Ebenso bedeuten die länderkundlichen Werke von Boesch (über den Nahen Osten oder die USA) und seine wirtschaftsgeographischen Bücher und Atlanten, wie das mit P. Hofer und G. Grosjean herausgegebene Bildwerk über die Schweizerstadt ${ }^{19}$, Impulse der Planung die kaum zu missen sind.

Leisteten somit die geographischen Institute der Zürcher Hochschulen unabhängig und gemeinsam (z. B. betätigten sich $\mathrm{H}$. Carol und der Schreibende mehrere Jahre zusammen in einer akademischen Studiengruppe für Landesplanung, der das vorhin erwähnte Buch und andere Untersuchungen entsprangen) von der Fachwelt anerkennend beurteilte Beiträge an die schweizerische Orts-, Regional- und Landesplanung, so blieben sie doch keineswegs allein. Vielmehr setzten sich in analoger Weise auch die Geographen der übrigen Hochschulen für sie ein, damit die Verwendung ihrer Disziplin ebenso eindrücklich belegend.

Um alphabetisch vorzugehen, reichen entsprechende Bestrebungen an der Universität Basel ebenfalls in die Anfänge ihres geographischen Instituts (1912) zurück. Sie bleiben mit den Namen G. Braun, H. Hassinger und F. Jaeger verknüpft, die in Vorlesungen wie in Publikationen für die angewandte Geo- graphie eintraten. Auch ihr Nachfolger P. Vosseler betonte immer wieder die praktische Bedeutung der Erdkunde für Volk und Land18. Eine neue Aera brach 1961 mit der Wahl von H. Annaheim zum Ordinarius für Geographie an. Er hatte schon früher, anfänglich vorwiegend geomorphologischen Problemen zugewandt, für den Einsatz der Geographie in die Praxis geworben. So setzte er 1953 als Zentralpräsident des Verbandes schweizerischer geographischer Gesellschaften bei dessen Statutenrevision unter Mitarbeit von E. Schwabe und dem Schreibenden die Einfügung eines Paragraphen «Förderung der Landschaftsplanung sowie landschaftsgestaltender und -schützender Maßnahmen» durch. Eigene grundlegende Beiträge insbesondere zur Planung von Basel wie namentlich seine Schrift «Die Raumgliederung des Hinterlandes von Basel» (1950) ${ }^{19}$ führten ihn zur unmittelbaren Verbindung mit der Planung, die er auch über die Tagespresse wirksam förderte. Als sein bisheriges Hauptwerk hat wohl der «Strukturatlas Regio-Nordwestschweiz -Oberelsaß-Südschwarzwald» (1966) gewürdigt zu werden. Mit ihm schuf er, unter Mitwirkung verschiedener Schüler (K. Eichenberger, W. Gallusser u.a.) nicht allein eine vorbildliche Grundlage für die Planung der Heimatregion, sondern ein erstmaliges Instrument völker- und länderverbindender Planung. Indem er dem geographischen Institut 1967 eine "Abteilung für geographische Grundlagenforschung der Basler Region» angliederte, gab er der Entwicklungsplanung ein Werkzeug, wie es nicht besser gewünscht werden kann'20.

An der Universität Bern hatten sich ebenfalls schon früh R. Zeller, später F. Nussbaum (1879-1966) und W. Staub (1886-1966) für die praktische Geographie verwendet. Ihr Nachfolger auf dem ordentlichen Lehrstuhl für Geographie, F. Gygax, (ab 1949) ist seit langem für angewandte Hydrologie tätig. Sie brachte ihn namentlich mit dem schweizerischen Kraftwerkbau in förderliche Verbindung, die umgekehrt seine morphologischen und hydrologischen Forschungen befruchtete, wie zahlreiche Dissertationen erkennen lassen21. Der seit 1954 die Kultur- und Wirtschaftsgeographie vertretende Kollege G. Grosjean, der das Erbe W. Staubs weiterführt, setzte sich seither erfolgreich für die Schaffung einer Abteilung für angewandte Geographie ein, die 1969 verwirklicht wurde. Auch machte er sich mittels eigener und Arbeiten seiner Schüler bei Regional- und Ortsplanungen sowie um den Landschaftsschutz verdient 22 . Beider Bestrebungen berechtigen zu besten Hoffnungen, zumal bereits Schüler (H. Heller, u. a.) Beschäftigung in der Planung gefunden haben.

Bei den welschschweizerischen Hochschulen ging nach verheißungsvollen, durch den dortigen Ordinarius für Geographie C. Burky (1887-1962) injizierten Anfängen die Führung in angewandter Geo- 
graphie in gewissem Sinne an die Universität Freiburg. Dort verwendete sich vor allem J.-L. Piveteau für sie. Er konnte sich hierbei der Hilfe von Lausanner Kollegen (L. Bridel, G. Barbier, M. Roten) versichern. Sie brachten, teilweise im Dienste des dortigen kantonalen Planungsamtes stehend, entsprechende praktische Erfahrungen mit, die zweifellos für die Geographenausbildung ebenso wertvoll wie nützlich sind 23 . Einer der Schüler Piveteaus, G. Schumacher, arbeitet überdies in der bernischen Stadtplanung.

An der Universität Genf hatte sich der frühere Vertreter der Anthropogeographie, C. Burky, der schon durch seine rege journalistische Tätigkeit eng mit dem wirtschaftlichen und politischen Leben liiert war, seit den Anfängen landesplanerischen Wirkens in der Schweiz um die Einbeziehung seines Faches bemüht. Als Mitglied verschiedener Planervereinigungen wie als Publizist erwarb er sich hierbei erhebliche Verdienste ${ }^{24}$. Sein Nachfolger, P. Guichonnet, sucht namentlich im Rahmen einer "Regio Genevensis» die Kontakte mit der Regionalplanung zu intensivieren ${ }^{25}$. Bei dieser Gelegenheit soll nicht vergessen werden, daß in Genf mehrere Jahre die "Matériaux pour l'étude des calamités» erschienen, welche eine wichtige Grundlage auch der Planung darstellen. An ihrer Äuffnung haben sich Geographen wie F. Montandon maßgebend beteiligt 26 .

In Lausanne hatte sich besonders C. Biermann (1875-1961) durch eigene Untersuchungen z. B. bezüglich des Waadtländer Bauernhauses und der Siedlungen des Kantons in den Dienst der Verwendung der erdkundlichen Forschung gestellt27. Sein Nachfolger H. Onde war vor allem bestrebt, neben theoretischen Arbeiten, die Schüler für die Regionalplanung $\mathrm{zu}$ interessieren28. Er war insofern durchaus erfolgreich, als Geographen wie L. Bridel, V. Ruffy u. a. sich in initiativer Weise diesem Metier zuwandten und seit längerem im Staatsdienst (Office vaudois de l'urbanisme) für die praktische Geographie wirken 29 .

In den letzten Jahren haben sich insbesondere dank der Einschaltung des Inhabers des Lehrstuhls für Ethnologie und Anthropogeographie, J. Gabus, auch in Neuenburg die Beziehungen zwischen Planung und Geographie zu verstärken begonnen. So beteiligt sich Gabus mit verschiedenen Mitarbeitern an der Planung der Region "Tielle», und er hofft, diese Studien in anderen Kantonsgebieten erweitern zu können ${ }^{30}$.

Die Hochschule St. Gallen für Wirtschafts- und Sozialwissenschaften schließlich besaß zwischen 1900 und 1940 in dem als Philosoph der Erdkunde bekannt gewordenen Wirtschaftsgeographen P. H. Schmidt einen nicht minder warmen Förderer angewandter Wissenschaft, der zudem lange Jahre hindurch als Sekretär des Industrievereins den prakti- schen Wert seines Faches bewies. Neben mehreren gewichtigen wirtschaftsgeographischen und methodologischen Büchern legt besonders sein letztes, "Auslandforschung", von seiner Überzeugung, Geographie sei zentrale Grundlage des Lebens, eindrucksvolles Zeugnis ab ${ }^{31}$. Das lange «Interregnum» nach seinem Rücktritt und Tod (1954) war leider weiterhin analogen Bestrebungen ungünstig. Doch sei immerhin an das Buch O. Widmers (1887 -1962) «Das Oberste Toggenburg» erinnert, das ganz der Planung und Erschließung dieses Gebietes gewidmet ist ${ }^{32}$. Der definitive Nachfolger, Erh. Winkler (seit 1964), der sich anfänglich besonders mit wirtschaftsgeographischen Untersuchungen im Orient beschäftigt hatte, wandte sich inzwischen Fragen seiner Wohnregion $\mathrm{zu}^{33}$.

Wenn von der Tätigkeit der Hochschulen die Rede ist, dürfen die Beiträge des ehemaligen Ordinarius für Kartographie der ETH, Ed. Imhofs, nicht vergessen werden. Er brachte nicht allein in Karten verschiedenster "Thematik» und in vorbildlichen Reliefs die geographische Theorie zu bildhaftem Ausdruck, sondern steuerte auch zahlreiche geographische Untersuchungen bei. Die wohl eindrücklichste Manifestation seines Wirkens ist in dem seit 1965 (1961) erscheinenden grundlegenden «Atlas der Schweiz» zu erblicken, bei welchem ihn neben zahlreichen andern Fachgenossen H.'Gutersohn als Mitredaktor unterstützt. Mit den vorgesehenen Planungskarten wird der Atlas überdies auch unmittelbar Beziehungen zur Praxis knüpfen.

Der Einsatz für die praktische Anwendung der Geographie kam nicht zuletzt in zahlreichen Dissertationen zum Ausdruck. Hier können jedoch nur wenige direkt auf die Gebietsplanung Bezug nehmende Beispiele namhaft gemacht werden, so W. Nigg: Das Schanfigg (Univ. Zürich, 1948); G. Camozzi: Il piano di Magadino (Univ. Zürich 1949); U. Haller: Zur Geographie der Region zwischen Zürich und Baden (ETH 1957); P. Widmer: Zur Geographie des Suhrentales (ETH, 1960); R.Wehrle: Agrargeographische Untersuchungen im Raume von Zürich (Univ. Zürich 1962); K. Hintermann: Zur Kulturgeographie des Baselbietes (ETH 1966); H. W. Muggli: Greater London und seine New Towns (Univ. Basel 1968) oder U. Eichenberger: Die Agglomeration Basel in ihrer raumzeitlichen Struktur (Univ. Basel 1968).

Die Anwendung der Geographie auf die Praxis blieb indessen keineswegs ein Privileg der Hochschulen. Auch zahlreiche außer ihnen tätige Geographen zählen zu ihren Förderern. Die sicher sympathischsten Beiträge wurden auf dem Gebiet des Landschaftsschutzes und der Landschaftspflege geleistet. Auf ihm ist vorab E. Egli zu nennen, dessen zahlreiche Vorträge und Publikationen im besten Sinne angewandte Geographie darstellen ${ }^{34}$. Weitere Namen wie H. Brunner, E. Erzinger, E. Gerber, H. 
Hofer, G. Grosjean, W. Nigg, E. Schwabe u. a. müssen an Stelle zahlreicher ungenannter Kollegen stehen, die der Verfasser unbeabsichtigt übersehen hat.

Erfreulich ist in diesem besonderen Zusammenhang, daß Geographen auch als vollamtliche Experten für Landschaftsschutz und Landschaftspflege ein dankbares, wenn auch kaum sehr sorgenloses Wirkungsfeld gefunden haben, so $\mathrm{M}$. Disteli (Kt. Aargau) ${ }^{35}$, neuerdings K. C. Ewald (Bund für Naturschutz). Nicht weniger wichtig, im Blick auf die 'Zukunft unseres Lebensraumes vielleicht sogar noch dringlicher, ist der freiwillige initiative Einsatz von Geographielehrern für Orts-, Regional- und Landesplanung. Ihnen ist W. Nigg ein nachahmenswertes Beispiel. Seit mehreren Jahren für die Landesplanung eintretend, organisiert er seit 1963 im Rahmen des neu eingerichteten Gruppenunterrichts der Kantonalen Oberrealschule Zürich Orientierungen über Landesplanung, bei welchem Praktiker mitwirken und während denen die Schüler auch Vorschläge auszuarbeiten haben ${ }^{36}$. Sein Kollege $\mathrm{H}$. Bernhard sekundierte ihn mit Ưbungen über Abwasserprobleme und Gewässerschutz. Es ist erfreulich, daß diese Vorbilder bereits an andern Schulen Nacheiferung fanden.

$\mathrm{Da}$ daneben auch andere Anwendungsgebiete der Geographie, wie etwa der staatsbürgerliche Unterricht mit Erfolg von Geographen gepflegt wurden, sei hier nur angedeutet ${ }^{37}$, da es dem Schreibenden vor allem darauf ankam, zu zeigen, inwieweit im Zentralbereich der Geographie, in der Landschaftsforschung und damit in der Landschaftsplanung und -pflege Möglichkeiten der Beteiligung bestehen und auch benützt worden sind, während ihm Politik, Wirtschaft oder Betätigungen im Bereiche von Landschaftselementen (angewandte Geomorphologie, Klimatologie usw.) höchstens sekundär in der Kompetenz des Geographen gelegene und daher nicht ungefährliche Anwendungsgebiete zu sein scheinen.

Der Rückblick hat wohl zu skizzieren vermocht, daß die sogenannte «angewandte» Geographie auch in der Schweiz eine nicht unbeachtliche Geschichte besitzt. Diese Geschichte schließt gleicherweise bemerkenswerte Möglichkeiten wie Verpflichtungen in sich, wenn - was die Geographen doch offenbar allgemein wünschen - ihrer Disziplin vermehrte Würdigung durch die Praxis zuteil werden soll. Solche Möglichkeiten dürften sich wohl namentlich in der gesteigerten Einschaltung in die Landschaftspflege bieten. Denn einerseits besteht nach wie vor ein empfindlicher Mangel an tüchtigen Landschafts- und Landesplanern, -gestaltern, -pflegern und -schützern. Andrerseits ist der Geograph, sofern für ihn die Landschaft das zentrale Objekt bedeutet, für deren Betreuung "von Natur» vorzugsweise prädestiniert. Dies gilt insbesondere deshalb, weil die der Geographie inhärente Hauptaufgabe: die gleichmäßige Berücksichtigung aller Elemente des Funktionsgefüges Landschaft von den übrigen Fachleuten, welche sich gleichfalls mit Landschaften befassen, noch nicht durchwegs und wesensgemäß erkannt zu sein scheint.

Zwei Momente freilich erschweren die unmittelbare und vermehrte Beteiligung des Geographen bei der Landschaftspflege (welcher hier Landschaftsplanung, -gestaltung und -schutz-, und zwar sowohl auf Natur- und Kulturlandschaften bezogen subsummiert sind). Das eine ist die noch zu wenig ausschließlich auf die praktische Befassung mit der Landschaft ausgerichtete Schulung des Geographen, der hiefür erheblich mehr technischer, rechtlicher und finanzwirtschaftlicher Kenntnisse bedarf als er sie sich bisher aneignete. Das andere Moment ist die Tatsache, daß - nicht zuletzt aus dem erstgenannten heraus - die praktische Funktion des Geographen von den Spezialisten kaum hinreichend erkannt wurde. $\mathrm{Da}$ andrerseits die meist den Kreisen der Gärtnerei entstammenden Landschaftsgestalter alle Anstrengungen unternehmen, entsprechende Einsichten zu verwirklichen, sollten auch die Geographen dahinter nicht zurückstehen. Hierbei ist nicht an eine einander gegenseitig ausschließende Konkurrenz zu denken. Vielmehr erschiene es wünschenswert und zweifellos auch erfolgverheißend, daß Geographen als Landschaftsforscher und Landschaftsplaner, -pfleger und -schützer sich vermehrt zusammenfänden, um dem an sich ebenso aktuellen wie dringenden Anliegen einer optimalen Landschaftspraxis gemeinsam zu dienen. Ein Anfang ist gemacht: Nachdem Geographie-Studierende schon ab 1961 an der ETH ein Schlußdiplom in Landesplanung erwerben konnten, haben sie seit 1967 Gelegenheit, mit Agronomen, Architekten, Bauingenieuren, Forstingenieuren, Juristen, Sozialökonomen und Soziologen zusammen die (viersemestrige) Nachdiplomausbildung für Landesplaner am Institut für Orts-, Regional- und Landesplanung (Direktor Prof. M. Rotach, Ausbildungsleiter Prof. J. Maurer) mitzumachen. Dabei sind auch Landschaftsplanung und -gestaltung Schulungsfächer. Obwohl die Beteiligung bisher allgemein beschränkt war, zeichnet sich damit doch eine bedeutsame Möglichkeit ab, die bereits von Geographen der Berner und Zürcher Hochschulen benützt wurde. Daß dennoch erhebliche weitere Auf- und Ausbauarbeit zu leisten ist, bedarf sicher keiner Erörterung. Der 1967 auf Initiative von W. Kündig gegründeten Schweizerischen Gesellschaft für angewandte Geographie erwächst gerade hieraus eine schöne Aufgabe.

Damit läßt sich abschließen. Es kann dies nicht treffender geschehen als mit den Worten des Jubilars, Prof. Gutersohns, anläßlich der Fünfzig-Jahr-Feier des Geographischen Instituts der Universität Basel 196238. Sie galten zwar der Geographie als Grund- 
lage der Orts-, Regional- und Landesplanung, haben aber im gleichen Maße Gültigkeit auch für die Landschaftspflege: «Für uns Bürger eines glücklichen Staatsgebildes ist die Landesplanung eine große Aufgabe. Sie soll ein Mittel, und zwar wahrscheinlich das wichtigste Mittel dazu sein, daß dieses Land uns Schweizern auch weiterhin ein glücklicher Lebensraum bleibt. Zur Verwirklichung einer erfolgreichen Landesplanung hat die Geographie zweifellos viel beizutragen. Es ist dieser Wissenschaft damit ein Arbeitszweig zugewiesen, der unsern Vorgängern kaum bewußt geworden war, sahen sie sich doch nicht derart fiebrigen landschaftlichen Entwicklungen gegenübergestellt wie wir. Seit einigen Jahren aber ist den Geographen diese neue zusätzliche Aufgabe bewußt geworden. Weichen wir ihr nicht aus, fassen wir die Mitwirkung bei der Landesplanung als eine Verpflichtung auf, eine Verpflichtung, deren Erfüllung dazu beiträgt, unsere Landschaften auch für die Zukunft gesund und schön zu erhalten".

\section{Benützte Quellen}

1 Für den Verfasser sind die Ausdrücke "Angewandte Geographie» und "Angewandte Wissenschaft» Paradoxa. Wissenschaft als Erkenntnissuche (Theorie) ist Korrelat zu Praxis, Anwendung. Angewandte Geographie ist keine "Graphie» oder Theorie (mehr), sondern Anwendung, Praxis. Andrerseits sind beide: Wissenschaft (Graphie) und Praxis (notwendig) aufeinander angewiesen, die eine ohne die andere nicht "möglich». Vgl. E. SzavaKovats: Zur Frage der angewandten Geographie. Geographica Helvetica 21, 1966, S. 122-131.

2 Die Geographie, ihre Geschichte, ihr Wesen und ihre Methoden. Breslau 1927, S. 156.

3 J. Kant: Physische Geographie. Ausgabe Rink, zit. nach Kants Werken, IX, Berlin 1923, S. 163.

4 A. a. O., S. 156.

5 Die pessimistische Beurteilung W. Hartkes. («Die Bedeutung der geographischen Wissenschaft in der Gegenwart» im Tagungsbericht des Deutschen Geographentages vom 22. bis 26. Mai 1961, Wiesbaden 1962, S. 113-131, bes. S. 115), die Geographie der Gegenwart habe "keine oder fast keine Bedeutung", braucht deshalb keineswegs unbedingt unterschrieben zu werden.

6 Bern und Zürich 1863.

7 J. Früh: Ausblicke auf die verschiedenen Gebiete der Geographie. Schweiz. Pädagog. Ztschr. 8, 1898, S. 4, sowie Geographie der Schweiz, St. Gallen 1930-1938.

8 Peterm. Geogr. Mitteil., 61, 1915, 12-18, 99$103,179-214$.

9 Die Innenkolonisation der Schweiz, Zürich 1919, S. $1-2$.
10 Vgl. 25 Jahre SVIL. Zürich 1944. Ferner: 50 Jahre SVIL. Zürich 1968.

11 Die geographischen Gesichtspunkte in der Rationalisierung des landwirtschaftlichen Wirtschaftsraumes in der Schweiz, Zürich 1940.

12 Vierteljahresschrift d. Naturf. Ges. in Zürich 83, 1938, S. 264-284.

13 Verhandl. d. Schweiz. Naturf. Ges. Locarno 1940, S. 231-232.

$14 \mathrm{Vgl}$. H. Gutersohn und E. Winkler: Zur Neuordnung der Landesplanung an der ETH. Plan 18, 1961, 69-81 sowie H. Gutersohn: Betrachtungen zur schweizerischen Straßenplanung. Die Autostr. 26, 1957, Nr. 7, und Institut de géographie de l'Ecole polytechnique fédérale. Cahiers de Géographie de Besançon 14, 1966, 67-68.

15 Plan 1, S. 59-63, vgl. auch «Beiträge zur Frage der geographischen Raumgliederung in der amerikanischen Literatur». Vierteljahrsschrift d. Naturf. Ges. in Zürich 91, 1946, S. 37-50 sowie "Institut de géographie de l'Université de Zurich». Cahiers de Géographie de Besançon 14, 1966, p. 65-66. 16 Geographica Helvetica 1, 1946, S. 185-245.

17 Zürich 1949.

$18 \mathrm{Vgl}$. $50 \mathrm{Jahre}$ geographisches Institut der Universität Basel. Regio Basiliensis III, 1962, S. 205-215. $19 \mathrm{Vgl}$. Vierteljahrshefte des Statist. Amtes des Kts. Baselstadt.

20 "Geographie». Verhandl. d. Naturf. Ges. Basel 78, 1967, 107-117 und "Secteurs de recherche à l'Institut de Géographie de Bâle». Cahiers de Géographie de Besançon 14, 1966, p. 49-50.

21 V. Binggeli: Prof. Dr. Fritz Gygax, Bern, 60jährig. Geographica Helvetica 22, 1968, S. 188. F. Gygax: Institut géographie de l'Université de Berne. Cahiers de Géogr. de Besançon 41, 1966, p.51—53.

$22 \mathrm{Vgl}$. etwa "Landschaft in Gefahr», Jhrb. vom Thuner- und Brienzersee 1940, S. 3-20 sowie "Institut de Géographie de l'Université de Berne. Cahiers de Géogr. d. Besançon 14, 1966, S. 52-53. 23 J.-L. Piveteau: Institut de géographie de Fribourg. Cahiers de Géogr. de Besançon 14, 1966, S. 55-56.

24 Tendance et perspectives du Plan d'aménagement national. Plan 16, 1959, p. 173-175.

25 P. Guichonnet: La Géographie à l'Université de Genève. Cahiers de Géogr. de Besançon 14, 1966, 57-58.

26 Matériaux pour l'étude des calamités. Genève 1924-37.

$27 \mathrm{Vgl}$. La Maison paysanne vaudoise. Lausanne 1946; Le Canton de Vaud. Lausanne 1952.

$28 \mathrm{H}$. Onde: L'institut de géographie de l'Université de Lausanne. Cahiers de Géographie de Besançon 14, 1966, 59-60.

29 L. Bridel: Les études en vue de l'aménagement régional et national. Cahiers de Géographie de Be- 
sançon 14, 1966, 31-44 (ein guter Überblick über die Regionalplanung der Schweiz und ihre Beziehung zur Geographie); vgl. auch H. Carol: Die Geographie als Grundlage praktischen Wirkens. Zur 50-Jahr-Feier des Vereins schweiz. Geographielehrer. Arau 1962, 41-45; ferner: E. Paillard: Le canton de Vaud et le plan d'aménagement régional. Le Globe 104, 1954, 93-122.

$30 \mathrm{~J}$. Gabus: Orientation générale de l'enseignement de la géographie et de l'ethnologie à l'Université de Neuchâtel. Cahiers de Géogr. de Besançon 14, 1966, 61-62.

31 Zürich 1945.

32 St. Gallen 1945.

33 E. Winkler: La géographie économique à l'Ecole des hautes etudes economiques et sociales de SaintGall. Cahiers de Géogr. de Besançon 14, 1966, 63 -64 .

34 E. Egli: Die Erhaltung der Landschaft in "Natur geplündert oder gehegt ». Zürich 1961, 19-32; Landschaft und Geographie. Mitteil. d. Naturf. Ges. Schaffhausen 28, 1967, 1-14; In Sorge um den Lebensraum. Jahrb. d. NHG 1968, SA 3-15.

35 Vgl. M. Disteli: Bedrohte helvetische Landschaft Neue Zürcher Ztg. 184, 1963, N. 2102; Natur- und Landschaftsschutz als Aufgabe unserer technisch geformten Zeit. Schweiz. Techn. Ztschr. 65, 577584; Was verlangen Landschaftsschutz und Land- schaftspflege von Erziehung, Wissenschaft und Technik. Natur und Mensch 11, 1969, 128-132. $36 \mathrm{Vgl}$. W. Nigg: Landesplanung an der Mittelschule. Geographica Helvetica 19, 1964, 192-196 sowie Landschaftsschutz und Landesplanung in Graubünden. Bündn. Jahrbuch 1968, 136-138; ferner O. Wernli: Soll und kann im Mittelschulunterricht die Landesplanung zur Sprache kommen? Plan 5, 1948, 195-196 sowie F. Schiesser: Geographische Übungen an der Oberstufe der Mittelschule. Geographica Helvetica XX, 1965, 34-36, und G. Grosjean: Schule und Landesplanung. Schweiz. Lehrerzeitung 112, 1967, 1267-1272.

37 Vgl. z. B. G. Rüetschi: Geographie und nationale Erziehung. St. Gallen 1916 oder K. Bösiger: Der Auftrag der Geographie für die staatsbürgerliche Erziehung. Regio Basiliensis IV, 1963, 101-121; E. Winkler: Länderkunde und Völkerverständigung. Friedenswarte 46, 1946, 121-135.

$38 \mathrm{H}$. Gutersohn in: Basler Beiträge z. Geogr. und Ethnologie 5, 1963, 7-24.

NB.: Die Liste ist raumeshalber völlig unvollständig. Namentlich hinsichtlich der Publikationen einzelner Autoren konnte sie nur einzelne wenige Beispiele bringen. Für kritische Hinweise dankt der Verfasser besonders Prof. Dr. W. Nigg.

\section{Schriftenverzeichnis Prof. Dr. H. Gutersohn}

zusammengestellt von Rosmarie Gschwend, Felix Kirchhofer, Ruedi Koblet und Max Rothenfluh

1929 In den Alpen. Der Schweizer Geograph, 6.Jg., S. 38/39, 54-57.

1930 Flußdichte, Untersuchungen in drei Gebieten der Schweiz. Vierteljahresschrift der Naturforschenden Gesellschaft Zürich, 75. Jg., S. 161 -

1932 Relief und Flußdichte, Diss. Universität Zürich, Prof. Flückiger, $90 \mathrm{~S}$.

1933 Geographische Skizzenblätter. Schweizerische Lehrerzeitung.

1934 Sonnenstrahlung und Bergschatten auf dem Gebiet der Stadt Zürich. Viertelj. Natf. Ges. Zch., 79. Jg., S. 1-11.
1935 Die Priele der Halligen. Der Schweizer Geograph, 12. Jg., S. 61-66.

1936 Ablation und Abfluß, Untersuchungen an Gletschern der Schweizer Alpen. Viertelj. Natf. Ges. Zch., 81. Jg., S. 177-198.

- Die Puszta Bugac. Zeitschrift Volkshochschule Zürich, 5. Jg., S. 231-236.

1937 Neuere deutsche Geographie. Zeitschr.Volkshochschule Zürich, 6. Jg., S. 49-54.

1938 Neue Niederschlagssammler. Der Schweizer Geograph, 15. Jg., S. 16-19.

- Die Naturlandschaft von Zürich. Mitteilungen der Geographisch-Ethnographischen Ge- 